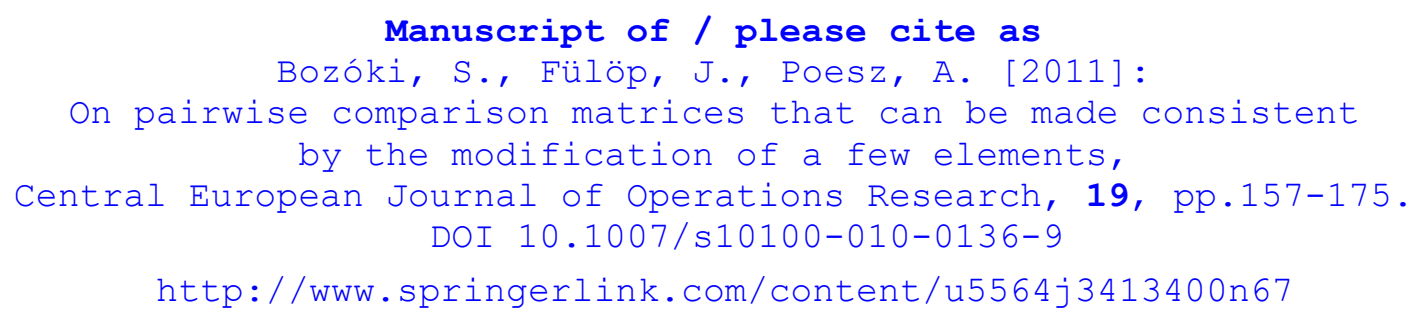

\title{
On pairwise comparison matrices that can be made consistent by the modification of a few elements
}

\author{
Sándor Bozóki ${ }^{1,2}$ • János Fülöp ${ }^{1,3}$ • Attila Poesz ${ }^{2}$
}

Received: date / Accepted: date

\begin{abstract}
Pairwise comparison matrices are often used in Multi-attribute Decision Making for weighting the attributes or for the evaluation of the alternatives with respect to a criteria. Matrices provided by the decision makers are rarely consistent and it is important to index the degree of inconsistency. In the paper, the minimal number of matrix elements by the modification of which the pairwise comparison matrix can be made consistent is examined. From practical point of view, the modification of 1, 2, or, for larger matrices, 3 elements seems to be relevant. These cases are characterized by using the graph representation of the matrices. Empirical examples illustrate that pairwise comparison matrices that can be made consistent by the modification of a few elements are present in the applications.
\end{abstract}

Keywords Multi-attribute decision making - Consistent pairwise comparison matrix . Graph representation of pairwise comparison matrices

\section{Introduction}

Tram tender of a city, facility location selection, purchase of a family car, selection among job offers, medical service selection or even the ranking of decathlon competitors are representative practical Multi-attribute Decision Making (MADM) problems. Formally, MADM is a prioritization or selection among a finite number of alternatives/actions that are characterized by a finite number of often non-commensurate and typically conflicting criteria.

In solving a multi-attribute decision problem, one needs to express the importances/weights of the attributes by numbers as well as the evaluations of the alternatives with respect to the attributes. The method of pairwise comparison matrices [11]

\footnotetext{
1 Laboratory on Engineering and Management Intelligence, Research Group of Operations Research and Decision Systems, Computer and Automation Research Institute, Hungarian Academy of Sciences; Mail: 1518 Budapest, P.O. Box 63, Hungary. Research was supported in part by OTKA grants K 60480, K 77420. E-mail: bozoki@sztaki.hu, fulop@sztaki.hu

2 Department of Operations Research, Corvinus University of Budapest. Mail: 1093 Budapest, Fővám tér 8., Hungary. E-mail: attila.poesz@stud.uni-corvinus.hu

3 Corresponding author
} 
is one of the most often used techniques. Consider $n$ items (weights of criteria, evaluations of the alternatives with respect to a criterion, or voting powers of individuals in group decision making) to be compared. The decision maker compares each pairs of the items and answers the question like 'How many times one is larger/better than the other one?'. An $n \times n$ matrix

$$
A=\left(\begin{array}{ccccc}
1 & a_{12} & a_{13} & \ldots & a_{1 n} \\
a_{21} & 1 & a_{23} & \ldots & a_{2 n} \\
a_{31} & a_{32} & 1 & \ldots & a_{3 n} \\
\vdots & \vdots & \vdots & \ddots & \vdots \\
a_{n 1} & a_{n 2} & a_{n 3} & \ldots & 1
\end{array}\right)
$$

is called pairwise comparison matrix if it is positive and reciprocal, i.e.,

$$
\begin{aligned}
& a_{i j}>0, \\
& a_{i j}=\frac{1}{a_{j i}},
\end{aligned}
$$

for $i, j=1, \ldots, n$.

A pairwise comparison matrix $A$ is consistent if it satisfies the transitivity property

$$
a_{i j} a_{j k}=a_{i k}
$$

for any indices $i, j, k,(i, j, k=1,2, \ldots, n)$. Otherwise, $A$ is inconsistent.

There is a number of methods for determining the weights from the pairwise comparison matrix filled in by the decision maker. Eigenvector method [11] and distance minimizing methods such as Least Squares Method [4,1,3] are just two of the basic ideas of the approximation of an inconsistent matrix by a consistent one. All weighting methods provide the same result for consistent matrices but not for inconsistent ones. However, in the paper, the focus is rather on the matrices that can be made consistent by modifying 1,2 or 3 of their elements than on weighting methods.

In real decision problems consistent matrices are rare but it is crucial to detect high inconsistencies. Contradictive responses of the decision maker may result in false outcomes. Nevertheless, the definition of the degree of inconsistency is not unique, there exist different measures and indices for it $[11,8,2]$. An alternative way is presented in the paper for finding the minimal number of elements in the pairwise comparison matrix by the modification of which it can be made consistent. Graph representation of pairwise comparison matrices $[5,7]$ is used in the paper as an efficient tool for a graphical interpretation of decision maker's preferences.

The paper is organized as follows. Graph representation of pairwise comparison matrices and its relations to consistency are presented in Section 2. In Section 3, a mixed $0-1$ program is defined for determining the minimal number of the elements whose modification can make a pairwise comparison matrix consistent. In Sections 4, 5 and 6 the cases of 1 element, 2 elements and 3 elements to modify, respectively, are discussed. Tests on empirical pairwise comparison matrices originated from real decisions are summarized in Section 7. 


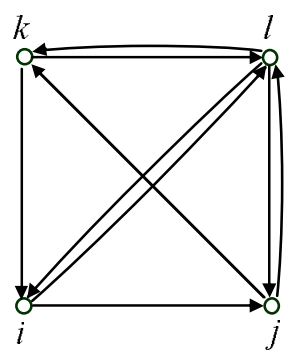

Fig. 1 The subgraph for the proof of Proposition 1

\section{Inconsistent triads}

Let $A$ be an $n \times n$ pairwise comparison matrix, and let

$$
\bar{A}=\log A
$$

denote the $n \times n$ matrix with

$$
\bar{a}_{i j}=\log a_{i j}, \quad i, j=1, \ldots, n .
$$

Then $A$ is consistent if and only if

$$
\bar{a}_{i j}+\bar{a}_{j k}+\bar{a}_{k i}=0, \forall i, j, k=1, \ldots, n .
$$

Introduce the directed graph $G=\{\mathcal{N}, \mathcal{A}\}$ where $\mathcal{N}=\{1, \ldots, n\}$ is the set of the nodes and $\mathcal{A}=\{(i, j) \mid i, j \in \mathcal{N}, i \neq j\}$ is the set of the edges. Let weights be associated with the edges of graph $G$, namely, weight $\bar{a}_{i j}$ with the edge $(i, j)$.

Let $i, j$ and $k$ be three different nodes of $G$. The cycle consisting of the three connecting edges $(i, j),(j, k),(k, i)$ is called a triad, denoted by $(i, j, k)$. The weight $w(i, j, k)$ of triad $(i, j, k)$ is defined by

$$
w(i, j, k)=\bar{a}_{i j}+\bar{a}_{j k}+\bar{a}_{k i} .
$$

It is clear that

$$
w(i, j, k)=w(j, k, i)=w(k, i, j)=-w(k, j, i)=-w(j, i, k)=-w(i, k, j),
$$

furthermore, matrix $A$ is consistent exactly when the weight of all triads is zero in the graph $G$ associated with $A$. A triad is called consistent if its weight is zero, otherwise, it is called inconsistent. We call the graph $G$ also consistent when all of its triads are consistent. In the sequel, when dealing with the number of the inconsistent triads, we consider the triads $(i, j, k),(j, k, i),(k, i, j),(k, j, i),(j, i, k),(i, k, j)$ as identical, and count them only once, since they are based on the same triple of nodes, and they are consistent or inconsistent simultaneously.

It is evident that for a consistent matrix $A$, the graph $G$ does not contain any inconsistent triad. However, as shown below, for an inconsistent $A$, the graph $G$ contains at least $n-2$ inconsistent triads.

Proposition 1. Let $(i, j, k)$ be an inconsistent triad. Then for any $l \in \mathcal{N} \backslash\{i, j, k\}$, at least one of the triads $(l, i, j),(l, j, k)$ and $(l, k, i)$ is inconsistent. 
Proof: It is easy to see, as shown in Figure 1, that

$$
w(l, i, j)+w(l, j, k)+w(l, k, i)=w(i, j, k) .
$$

Note that Figure 1 shows the subgraph of $G$ consisting only of the nodes and edges needed in the proof. Since $w(i, j, k) \neq 0$, at least one of the other three triads must have a nonzero weight.

Since the node $l \in \mathcal{N} \backslash\{i, j, k\}$ can be chosen in $n-3$ ways, and $(i, j, k)$ is inconsistent, we obtain directly:

Corollary 1. If $A$ is inconsistent, then $G$ contains at least $n-2$ inconsistent triads

Corollary 2. If $A$ is inconsistent, then for any $i \in \mathcal{N}, G$ contains an inconsistent triad $(i, j, k)$.

A direct practical application of Corollary 2 is the following: when we want to check whether the pairwise comparison matrix $A$ is consistent or not, instead of checking the consistency of $\left(\begin{array}{l}n \\ 3\end{array}\right)$ triads, it is enough to do that for $\left(\begin{array}{c}n-1 \\ 2\end{array}\right)$ triads.

Corollary 2 has the further meaning that the inconsistency of a triad spreads over, namely, any alternative (or criterion) taking role in the pairwise comparison cannot elude the effect of the inconsistency among any three alternatives (or criterion). This is why it is so difficult to find a cause of the inconsistency in a pairwise comparison matrix.

\section{The minimal number of elements to be modified}

It can happen in the practice that the person who performs the pairwise comparisons works basically in a precise and consistent way, and makes errors only in a few cases. If the classic eigenvector or optimization techniques mentioned and referred in the Introduction are applied in this case, then in the consistent pairwise comparison matrix obtained as an approximation by these techniques, the earlier correct pairwise comparison values may be destroyed. Furthermore, the alternatives may get merit weights not reflecting the real situation at all.

An inconsistent pairwise comparison matrix can be made consistent by modifying $K$ of its elements (and their reciprocals) if and only if it can be obtained from a consistent matrix by modifying $K$ of the elements (and their reciprocals) in the latter matrix. In the following, when we speak about the modification of an element in a pairwise comparison matrix, the appropriate modification of the reciprocal is also reckoned in, even if it is not mentioned explicitly. The modification concerns, of course, only the off-diagonal elements since every element is 1 in the diagonal of a pairwise comparison matrix.

In the following we present some tools to elicit whether an inconsistent pairwise comparison matrix can be made consistent by modifying a few $(1,2$ or 3$)$ of its elements. If the answer is affirmative, it may be worthwhile calling the attention of the person performing the pairwise comparisons to this fact. It may happen that he made indeed mistakes, and he is disposed to reconsider those values. However, of course, he may insist on those values, and other, classic tools can be applied for handling the inconsistent pairwise comparison matrix in the further steps.

Two approaches will be proposed. The first one constructs a mixed 0-1 programming problem to answer the question how can an inconsistent pairwise comparison 
matrix be made consistent by modifying the minimal number of its elements. The second approach is based on elementary, graph theoretic analysis of the graph $G$.

Assume that an $M \geq 1$ is given serving as an upper bound on the values of the elements in the original and the modified pairwise comparison matrices, i.e. we have

$$
a_{i j} \leq M, i, j=1, \ldots, n
$$

for the elements of $A$, and we want the modified matrix also with this property. In the practice, this is not a serious restriction since an interval of the reasonable values is usually known. In the Analytic Hierarchy Process $M$ is set to 9 , however, it is defined only for the elements given by the decision maker. The role of $M$ related to inconsistency indices is discussed in [2]. Let $\bar{M}=\log M$, this is an upper bound on the absolute value of the logarithms of the original and the modified elements. Consider the following optimization problem:

$$
\begin{array}{rlrl}
\min \sum_{i=1}^{n-1} \sum_{j=i+1}^{n} y_{i j} & \\
\text { s.t. } \quad x_{i j}+x_{j k}+x_{k i} & =0, & \\
x_{i j} & =-x_{j i}, & & 1 \leq i<j<k \leq n, \\
-\bar{M} \leq x_{i j} \leq \bar{M}, & & 1 \leq i<j \leq n, \\
-2 \bar{M} y_{i j} \leq x_{i j}-\bar{a}_{i j} & \leq 2 \bar{M} y_{i j}, & 1 \leq i<j \leq n, \\
y_{i j} & \in\{0,1\}, & 1 \leq i<j \leq n,
\end{array}
$$

where $x_{i j}, i, j=1, \ldots, n, i \neq j$, are continuous variables and denote the logarithms of the elements in the modified matrix, $y_{i j}, i=1, \ldots, n-1, j=i+1, \ldots, n$, are binary variables meaning that the modification is allowed in the position $(i, j)\left(\right.$ then $\left.y_{i j}=1\right)$ or not (then $\left.y_{i j}=0\right)$. The following statement is evident:

Proposition 2. The optimal value of problem (2) gives the minimal number of the elements that can be modified to make the pairwise comparison matrix $A$ consistent assuming (1) for $A$ and requiring (1) for the modified matrix.

If we only want to know whether the matrix $A$ can be made consistent by modifying at most $K$ of its elements, then the constraint

$$
\sum_{i=1}^{n-1} \sum_{j=i+1}^{n} y_{i j} \leq K
$$

is to be added to (2), and it is enough to search only for a feasible solution of (2)-(3).

The practical computational application of the above approach necessitates that an optimization software capable to solve problems (2) or (2)-(3) be callable from the decision support system. An integer programming method of general purpose can solve problems (2) and (2)-(3) with $\left(\begin{array}{l}n \\ 2\end{array}\right)$ binary variables in an exponential number of steps.

An advantage of the graph theoretic approach to be proposed below is that it does not necessitates the application of an optimization tool, and it is easy to implement. Constraint (1) that served basically to establish the mixed-binary program (2) can also be omitted. Furthermore, for a given $K$, polynomial algorithms can be applied contrary to exponential algorithms needed to solve problems (2) and (2)-(3). 


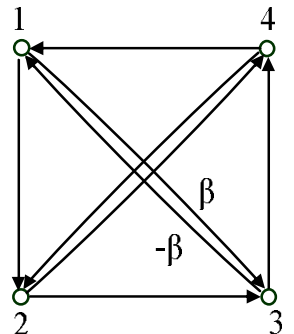

Fig. 2 The subgraph for the proof of Proposition 3 in case of $n=4$

\section{The case of single modification}

A special case of problem (2) is when an appropriate change of a single element leads to a consistent matrix.

Proposition 3. An inconsistent pairwise comparison matrix $A$ can be made consistent by modifying a single element if and only if the corresponding graph $G$ contains exactly $n-2$ inconsistent triads. If $n \geq 4$, then the modification, if any, is unique.

Proof: To prove the necessity, assume that $A$ can be obtained by modifying a single element of a consistent matrix. If we modify a single element of consistent matrix, then the weight of the corresponding edge (and of the opposite edge) is also modified. This edge is exactly in $n-2$ triads of the graph, neglecting the direction of the edges. The modification of the weight of the considered edge implies the modification of the weight of $n-2$ triads from zero to nonzero, the weight of any other triad does not change however. Consequently, the graph $G$ obtained by modifying a single element contains $n-2$ inconsistent triads.

To prove the sufficiency, assume that graph $G$ contains $n-2$ inconsistent triads. In case of $n=3$, it is trivial that $A$ can be made consistent by a suitable modification of any of its off-diagonal elements. The case of $n=4$ is also easy to handle. Then $N=\{1,2,3,4\}$, there are four triads in the graph, two of them are inconsistent, and two of them are consistent. We can assume, without loss of generality, that the triads $(1,2,3)$ and $(1,3,4)$ are inconsistent, and the triads $(2,4,1)$ and $(2,3,4)$ are consistent

(Figure 2). Then

$$
\bar{a}_{2,4}+\bar{a}_{4,1}+\bar{a}_{1,2}=0, \quad \bar{a}_{2,3}+\bar{a}_{3,4}+\bar{a}_{4,2}=0 .
$$

By adding up the two equalities and using $\bar{a}_{i j}=-\bar{a}_{j i}, i, j=1, \ldots, 4$, we get

$$
\bar{a}_{1,2}+\bar{a}_{2,3}=\bar{a}_{1,4}+\bar{a}_{4,3} .
$$

Let $\beta$ denote the value of the both sides in the previous equality. Modify the weight of the edge $(1,3)$ as follows:

$$
\bar{a}_{1,3}=\beta, \bar{a}_{3,1}=-\beta .
$$

Now the triads $(1,2,3)$ and $(1,3,4)$ are already consistent, but the modification does not concern the earlier consistency of the triads $(2,4,1)$ and $(2,3,4)$.

We turn now to the case of $n \geq 5$. Consider an inconsistent triad $(i, j, k)$, and let $\alpha=w(i, j, k)$. Since the graph $G$ contains $n-2$ inconsistent triads, it follows from Proposition 1 that for any $l \in \mathcal{N} \backslash\{i, j, k\}$ exactly one of the triads $(l, i, j),(l, j, k)$ 


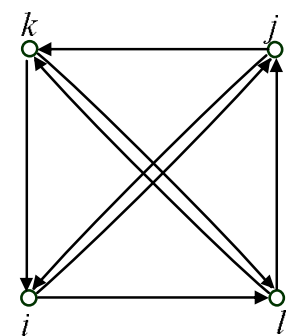

Fig. 3 The subgraph for the proof of Proposition 3 in case of $n \geq 5$

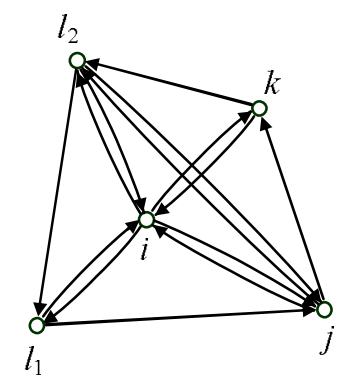

Fig. 4 The subgraph to show that the same edge of $(i, j, k)$ appears in the inconsistent triads

and $(l, k, i)$ is inconsistent. We show that the weight of this inconsistent triad is also $\alpha$. Assume that for an $l \in \mathcal{N} \backslash\{i, j, k\}$ the triad $(l, i, j)$ is inconsistent (Figure 3). Then the triads $(l, j, k)$ and $(l, k, i)$ are consistent, and adding up the equalities

$$
\bar{a}_{l j}+\bar{a}_{j k}+\bar{a}_{k l}=0, \quad \bar{a}_{l k}+\bar{a}_{k i}+\bar{a}_{i l}=0
$$

and rearranging them, we get

$$
\bar{a}_{j k}+\bar{a}_{k i}=\bar{a}_{j l}+\bar{a}_{l i}
$$

thus

$$
\bar{a}_{i j}+\bar{a}_{j k}+\bar{a}_{k i}=\bar{a}_{l i}+\bar{a}_{i j}+\bar{a}_{j l}=\alpha .
$$

We show now that for any $l \in \mathcal{N} \backslash\{i, j, k\}$, always the same edge of the triad $(i, j, k)$ appears in the inconsistent triad obtained by including the node $l$. Assume contrarily that for an $l_{1} \in \mathcal{N} \backslash\{i, j, k\}$ the triad $\left(l_{1}, i, j\right)$ is inconsistent, and for another $l_{2} \in \mathcal{N} \backslash\{i, j, k\}$ the triad $\left(l_{2}, k, i\right)$ is inconsistent (Figure 4$)$. Then $\left(l_{2}, j, k\right)$ is consistent, and the triad $\left(j, l_{2}, l_{1}\right)$ is also consistent since any inconsistent triad must have an edge from those of $(i, j, k)$. Summing up the weights of $\left(l_{2}, j, k\right)$ and $\left(j, l_{2}, l_{1}\right)$ we get

$$
\bar{a}_{j k}+\bar{a}_{k l_{2}}+\bar{a}_{l_{2} l_{1}}+\bar{a}_{l_{1} j}=0 .
$$

The triad $\left(i, l_{2}, l_{1}\right)$ is consistent, i.e. $w\left(i, l_{2}, l_{1}\right)=0$, furthermore, $w(i, j, k)=\alpha$ and $w\left(l_{1}, j, i\right)=w\left(l_{2}, i, k\right)=-\alpha$. As shown in Figure 4, summing up the weights of triads $\left(i, l_{2}, l_{1}\right),\left(l_{1}, j, i\right),(i, j, k)$ and $\left(l_{2}, i, k\right)$ we get

$$
\bar{a}_{j k}+\bar{a}_{k l_{2}}+\bar{a}_{l_{2} l_{1}}+\bar{a}_{l_{1 j}}=-\alpha,
$$


that, because of $\alpha \neq 0$, contradicts (4).

For the sake of simplicity assume that for any $l \in \mathcal{N} \backslash\{i, j, k\}$ the triad $(l, i, j)$ is inconsistent. The weight of the triads $(l, i, j)$ is just $w(i, j, k)=\alpha$. Modify the value of $\bar{a}_{i j}$ to $\bar{a}_{i j}-\alpha$. By this modification, the weights of the triads based on the edge $(i, j)$ become zero, but the weights of the originally consistent triads do not change. Consequently, by the appropriate modification of the weight of edge $(i, j)$, as well as by modifying the elements $a_{i j}$ (and $a_{j i}$ ), matrix $A$ has been made consistent.

In the case of $n \geq 4$ the uniqueness of the modification comes from the fact that only that edge $(i, j)$ found above appears in all the $n-2$ inconsistent triads. The necessary modification of the edge $(i, j)$ is also unique. If the weight of any other edge was modified, that would leave the weight of at least one of the $n-2$ inconsistent triads unchanged.

Remark 1. It is easy to see that checking whether the number of the inconsistent triads is $n-2$ or not can be performed with $O\left(n^{3}\right)$ operations. If the condition holds, the edge to be modified and how to modify can be obtained immediately from the common edge and the same weight of any pair of inconsistent triads.

Proposition 4. Let $A$ be a pairwise comparison matrix obtained from a consistent pairwise comparison matrix by modifying $K$ elements (and their reciprocals). Then the graph $G$ associated with $A$ contains at most $K(n-2)$ inconsistent triads.

Proof: If we start from a graph associated with a consistent matrix, and in each of $K$ steps we modify the weight of an edge, then the weight of $n-2$ triads is modified in every step. The weight of a triad can change even in more than one step, but altogether, the weight of at most $K(n-2)$ triads can change. Since every triad was consistent at starting, $G$ can contain at most $K(n-2)$ inconsistent triads.

Contrary to the case of $K=1$, when exactly $n-2$ inconsistent triads are in the obtained graph, in the case of $K>1$ the number of the inconsistent triads depends on the connection of the modified edges and the relations among the modifications, too. It is easy to see that when the modified edges are independent, i.e. the edges have no common nodes, then the number of inconsistent triads is just $K(n-2)$. However, if some of the edges are connected, then the effects of the modifications can extinguish each other so the weight of a modified triad may finally become zero. It is shown in [9] that in case of $K=2$ the number of the inconsistent triads can vary between $K(n-2)-2$ and $K(n-2)$, and in case of $K=3$ between $K(n-2)-6$ and $K(n-2)$. The reader can easily verify these findings by enumerating the possible dispositions of the modified edges. It can even happen that after modifying $K$ elements, the matrix remains consistent as shown in the example below.

Example 1. Let $n \geq 3$, and consider the $n \times n$ pairwise comparison matrix defined by

$$
a_{i j}= \begin{cases}\alpha, & i=1 ; j=2, \ldots, n, \\ 1 / \alpha, & j=1 ; i=2, \ldots, n, \\ 1, & \text { otherwise }\end{cases}
$$

where $\alpha>0$ is arbitrary. It is easy to see that this matrix is consistent. Modifying the $n-1$ elements with value $\alpha$ to another value of $\alpha$, and modifying the reciprocals, too, another consistent pairwise comparison matrix is obtained.

In the light of Propositions 3 and 4, it may arise the conjecture that an inconsistent pairwise comparison matrix $A$ can be made consistent by modifying at most $K$ elements 


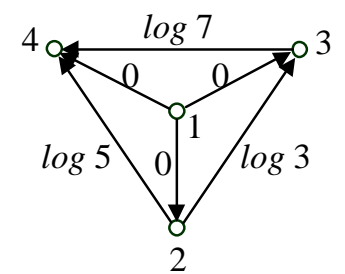

Fig. 5 The subgraph of Example 2

if and only if the associated graph $G$ contains at most $K(n-2)$ inconsistent triads. This conjecture is however not true as shown in the next example.

Example 2. Let $n=4$ and

$$
A=\left(\begin{array}{llll}
1 & 1 & 1 & 1 \\
1 & 1 & 3 & 5 \\
1 & 1 / 3 & 1 & 7 \\
1 & 1 / 5 & 1 / 7 & 1
\end{array}\right)
$$

All the four triads of the graph $G$ associated with $A$ are inconsistent, in addition, their weights are different (Figure 5). By Proposition 3, it is clear that $A$ cannot be made consistent by modifying a single element. Since for $K=2$ we have $K(n-2) \leq 4$, if the conjecture was true, then $A$ would be made consistent by modifying two elements. However, after having modified the weight of any of the edges of $G$, at least three inconsistent triads remain, and they cannot be corrected by modifying the weight of a further edge.

As the example above shows, merely the number of the inconsistent triads does not yield a sufficient condition. The connection of the inconsistent triads, their weights and the relations among them are also to be taken into account. Proposition 3 is rephrased in terms of this remark, and its proof comes directly from that of Proposition 3.

Proposition 5. An inconsistent pairwise comparison matrix $A$ can be made consistent by modifying a single element if and only if there exists an edge $(i, j)$ in the associated graph $G$ such that the weight of all triads $(l, i, j), l \in \mathcal{N} \backslash\{i, j\}$ is the same nonzero value, and all the other triads are consistent. For the edge $(i, j)$ with this property, the modification is unique. If $n \geq 4$, then there exits at most one edge $(i, j)$ with this property.

\section{Modification of two elements}

The phrasing of the proposition concerning the modification of the weight of two edges is similar to that of Proposition 5, it is however more involved since the connection of the two edges is also to be taken into account. Namely, the two edges are either independent (Figure 6(a)) or are connected in a common node (Figure 6(b)). It is easy to see that in case of $n=3$ only the layout of Figure 6(b) can occur, furthermore, any inconsistent matrix $A$ can be made consistent by modifying two elements in an infinite number of ways. 


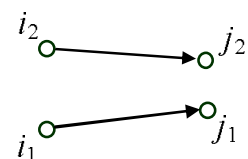

(a)

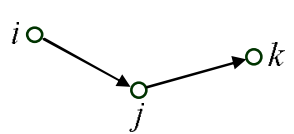

(b)

Fig. 6 The possible dispositions of two edges

Proposition 6. An inconsistent pairwise comparison matrix $A$ can be made consistent by modifying two elements if and only if exactly one of the following two conditions holds in the graph $G$ associated with $A$ :

1. There are two independent edges $\left(i_{1}, j_{1}\right)$ and $\left(i_{2}, j_{2}\right)$, and nonzero values $\alpha_{1}$ and $\alpha_{2}$ such that $w\left(l, i_{1}, j_{1}\right)=\alpha_{1}$ for all $l \in \mathcal{N} \backslash\left\{i_{1}, j_{1}\right\}, w\left(l, i_{2}, j_{2}\right)=\alpha_{2}$ for all $l \in \mathcal{N} \backslash\left\{i_{2}, j_{2}\right\}$, and all other triads are consistent.

2. There are two connected edges $(i, j)$ and $(j, k)$, and nonzero values $\alpha_{1}$ and $\alpha_{2}$ such that $w(l, i, j)=\alpha_{1}$ and $w(l, j, k)=\alpha_{2}$ for all $l \in \mathcal{N} \backslash\{i, j, k\}, w(i, j, k)=\alpha_{1}+\alpha_{2}$, and all other triads are consistent.

If $n \geq 4$, then for any pair of edges fulfilling conditions 1 or 2 , the modification of the weights of the edges that makes the graph $G$ consistent is unique. If $n \geq 5$, then there exists at most one pair of edges fulfilling condition 1 . If $n \geq 6$, then there exists at most one pair of edges fulfilling condition 2 .

Proof: First, assume that $A$ can be made consistent by modifying two elements. The two corresponding edges in graph $G$ are either the independent $\left(i_{1}, j_{1}\right)$ and $\left(i_{2}, j_{2}\right)$, or the connected $(i, j)$ and $(j, k)$. Assume that in order to make $A$ consistent we add a nonzero $\beta_{1}$ to $\bar{a}_{i_{1} j_{1}}$ and a nonzero $\beta_{2}$ to $\bar{a}_{i_{2} j_{2}}$ in the first case, and a nonzero $\beta_{1}$ to $\bar{a}_{i j}$ and a nonzero $\beta_{2}$ to $\bar{a}_{j k}$ in the second case. Consequently, in the first case, the weight of triad $\left(l, i_{1}, j_{1}\right)$ is modified by adding $\beta_{1}$ to it for all $l \in \mathcal{N} \backslash\left\{i_{1}, j_{1}\right\}$, and the weight of triad $\left(l, i_{2}, j_{2}\right)$ is modified by adding $\beta_{2}$ to it for all $l \in \mathcal{N} \backslash\left\{i_{2}, j_{2}\right\}$. Similarly, in the second case, the weights of triads $(l, i, j)$ and $(l, j, k)$ are modified by adding $\beta_{1}$ and $\beta_{2}$, respectively, to them for all $l \in \mathcal{N} \backslash\{i, j, k\}$, and the weight of triad $(i, j, k)$ is modified by adding $\beta_{1}+\beta_{2}$ to it. Since the weight of any triad is zero in the modified graph, it is evident that with $\alpha_{1}=-\beta_{1}$ and $\alpha_{2}=-\beta_{2}$, condition 1 holds in the first case, and condition 2 holds in the second case.

The sufficiency is trivial. If condition 1 holds, we add $-\alpha_{1}$ to $\bar{a}_{i_{1} j_{1}}$ and $-\alpha_{2}$ to $\bar{a}_{i_{2} j_{2}}$. Similarly, if condition 2 holds, we add $-\alpha_{1}$ to $\bar{a}_{i j}$ and $-\alpha_{2}$ to $\bar{a}_{j k}$, and all triads become consistent.

We show now that at most one of conditions 1 and 2 can hold. Assume contrarily that both conditions hold. This means that $G$ can be obtained from a consistent graph by modifying the weights of independent edges $\left(i_{1}, j_{1}\right)$ and $\left(i_{2}, j_{2}\right)$, and can be made consistent by modifying the weights of two connected edges $(i, j)$ and $(j, k)$. However, the four triads based on $i_{1}, j_{1}, i_{2}$ and $j_{2}$ are inconsistent, and it is easy to see that all of them cannot be made consistent by modifying the weights of two connected edges.

It follows also easily from the proof above that if $n \geq 4$ and a pair of edges fulfills conditions 1 or 2 , then the modification of the weights of the two edges to make $G$ consistent is unique. 
Let $n \geq 5$, and consider a pair of edges fulfilling condition 1. Assume that there is another pair of edges with this property. We can assume without loss of generality that the first pair is the one constructed in the proof of sufficiency, i.e. the one in Figure 6(a), furthermore, the edge $\left(i_{1}, j_{1}\right)$ is in the first pair but is not in the second one. Since $n \geq 5$, there is a node $l \in \mathcal{N}$ different from the endpoints of the edges $\left(i_{1}, j_{1}\right)$ and $\left(i_{2}, j_{2}\right)$. The three triads $\left(l, i_{1}, j_{1}\right),\left(i_{2}, i_{1}, j_{1}\right)$ and $\left(j_{2}, i_{1}, j_{1}\right)$ are inconsistent. It is evident that these three triads cannot be made consistent by modifying the weights of the second pair of edges since $\left(i_{1}, j_{1}\right)$ is not in the second pair.

Let $n \geq 6$, and now consider a pair of edges fulfilling condition 2. Again, assume that there is another pair of edges with this property. We can assume without loss of generality that the first pair is the one constructed in the proof of sufficiency, i.e. the one in Figure 6(b), furthermore, the edge $(i, j)$ is in the first pair but is not in the second one. Since $n \geq 6$, there exist three different nodes $l_{1}, l_{2}$ and $l_{3}$ also different from $i, j$ and $k$. The triads $\left(l_{1}, i, j\right),\left(l_{2}, i, j\right)$ and $\left(l_{3}, i, j\right)$ are inconsistent, and they cannot be made consistent by modifying the weights of the second pair of edges since $(i, j)$ is not in the second pair.

Remark 2. If $n=4$, then the number of the pairs of edges fulfilling conditions 1 or 2 may not be unique. This means that an inconsistent pairwise comparison matrix $A$ can be altered into different consistent forms by modifying two elements (and their reciprocals). For example, condition 1 holds for the graph $G$ associated with the inconsistent pairwise comparison matrix

$$
\left(\begin{array}{cccc}
1 & a & 1 & 1 \\
1 / a & 1 & 1 & 1 \\
1 & 1 & 1 & 1 / a \\
1 & 1 & a & 1
\end{array}\right)
$$

where $a \neq 1$, and (5) can be altered into the different consistent form

$$
\left(\begin{array}{llll}
1 & 1 & 1 & 1 \\
1 & 1 & 1 & 1 \\
1 & 1 & 1 & 1 \\
1 & 1 & 1 & 1
\end{array}\right) \quad \text { and } \quad\left(\begin{array}{cccc}
1 & a & a & 1 \\
1 / a & 1 & 1 & 1 / a \\
1 / a & 1 & 1 & 1 / a \\
1 & a & a & 1
\end{array}\right)
$$

by modifying two elements (and their reciprocals). Similarly, condition 2 holds for the graph $G$ associated with the inconsistent pairwise comparison matrix

$$
\left(\begin{array}{cccc}
1 & a & 1 & b \\
1 / a & 1 & 1 & 1 \\
1 & 1 & 1 & 1 \\
1 / b & 1 & 1 & 1
\end{array}\right)
$$

where $a \neq 1, b \neq 1, a \neq b$, and (6) can be altered into the different consistent forms

$$
\left(\begin{array}{llll}
1 & 1 & 1 & 1 \\
1 & 1 & 1 & 1 \\
1 & 1 & 1 & 1 \\
1 & 1 & 1 & 1
\end{array}\right), \quad\left(\begin{array}{cccc}
1 & a & a & a \\
1 / a & 1 & 1 & 1 \\
1 / a & 1 & 1 & 1 \\
1 / a & 1 & 1 & 1
\end{array}\right) \quad \text { and } \quad\left(\begin{array}{cccc}
1 & b & b & b \\
1 / b & 1 & 1 & 1 \\
1 / b & 1 & 1 & 1 \\
1 / b & 1 & 1 & 1
\end{array}\right)
$$

by modifying two elements (and their reciprocals).

There are four inconsistent triads in (5) and three in (6), thus, according to Proposition 3 , neither (5) or (6) can be made consistent by modifying a single element and 
its reciprocal. For $n=4$, it can be shown that the maximal number of the different pairs of edges fulfilling condition 1 is two, and this number is three for condition 2 . The proof, based on enumeration of the possible cases and simple arithmetics, is left to the reader.

Remark 3. If $n=5$, then the number of the pairs of edges fulfilling condition 2 may not be unique. For example, condition 2 holds for the graph $G$ associated with the inconsistent pairwise comparison matrix

$$
\left(\begin{array}{ccccc}
1 & a & a & 1 & 1 \\
1 / a & 1 & 1 & 1 & 1 \\
1 / a & 1 & 1 & 1 & 1 \\
1 & 1 & 1 & 1 & 1 \\
1 & 1 & 1 & 1 & 1
\end{array}\right)
$$

where $a \neq 1$, and (7) can be altered into the different consistent forms

$$
\left(\begin{array}{lllll}
1 & 1 & 1 & 1 & 1 \\
1 & 1 & 1 & 1 & 1 \\
1 & 1 & 1 & 1 & 1 \\
1 & 1 & 1 & 1 & 1 \\
1 & 1 & 1 & 1 & 1
\end{array}\right) \quad \text { and } \quad\left(\begin{array}{ccccc}
1 & a & a & a & a \\
1 / a & 1 & 1 & 1 & 1 \\
1 / a & 1 & 1 & 1 & 1 \\
1 / a & 1 & 1 & 1 & 1 \\
1 / a & 1 & 1 & 1 & 1
\end{array}\right)
$$

by modifying two elements (and their reciprocals). Since there are four inconsistent triads in (7), it cannot be made consistent by modifying a single element and its reciprocal. For $n=5$, it can be shown that the maximal number of the different pairs of edges fulfilling condition 2 is two. The proof is left again to the reader.

Remark 4. To perform the operations according to Proposition 6, we have first to prepare the list of the inconsistent triads. This can be done with $O\left(n^{3}\right)$ operations. If the number of the inconsistent triads is less than $2(n-2)-2$ or greater than $2(n-2)$, then it is sure that there is not any pair of edges fulfilling conditions 1 or 2 of Proposition 6. Otherwise, from the list of the edges appearing in the list of the inconsistent triads, we can prepare a list of $O\left(n^{2}\right)$ pairs of edges as candidates to fulfill conditions 1 or 2 . For each of these pairs, we can check condition 1 if the two edges are independent, or condition 2 if they are connected with $O(n)$ operations. To check that only triads based on at least one of the two edges can be found in the list of the inconsistent triads, $O(n)$ further operations are needed. Altogether, the pairs of edges fulfilling conditions 1 or 2 , if any, and how to modify can be determined with $O\left(n^{3}\right)$ operations. Remember, however, that even if the number of the inconsistent triads is $2(n-2)-2,2(n-2)-1$ or $2(n-2)$, it may happen that there is not any pair of edges fulfilling conditions 1 or 2 of Proposition 6, as shown in Example 2.

\section{Modification of three elements}

The case of $K=3$, i.e. the investigation whether an inconsistent matrix $A$ can be made consistent by modifying three of its elements (and their reciprocals) is more complicated than the cases of $K=1$ and $K=2$. In the graph $G$, there are five different types of the 


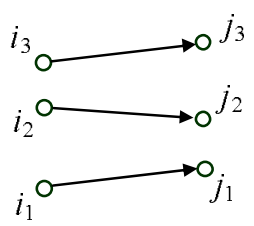

(a)

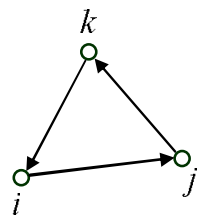

(d)

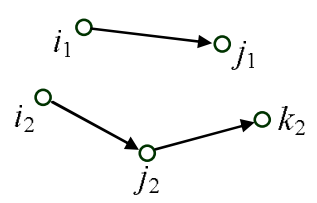

(b)

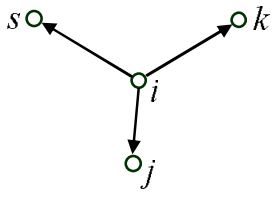

(e)

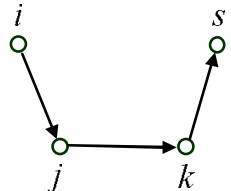

(c)

Fig. 7 The possible dispositions of three edges

connection and disconnection of the three edges as shown in Figure 7. Thus, following Proposition 6, five possible conditions are to be investigated.

Proposition 7. An inconsistent pairwise comparison matrix $A$ can be made consistent by modifying three elements if and only if at least one of the following five conditions holds in the graph $G$ associated with $A$ :

1. There are independent edges $\left(i_{t}, j_{t}\right)$ and nonzero values $\alpha_{t}, t=1,2,3$, such that $w\left(l, i_{t}, j_{t}\right)=\alpha_{t}$ for all $l \in \mathcal{N} \backslash\left\{i_{t}, j_{t}\right\}, t=1,2,3$, and all other triads are consistent.

2 . There are edges $\left(i_{1}, j_{1}\right),\left(i_{2}, j_{2}\right),\left(j_{2}, k_{2}\right)$, where $\left|\left\{i_{1}, j_{1}, i_{2}, j_{2}, k_{2}\right\}\right|=5$, and nonzero values $\alpha_{1}, \alpha_{2}, \alpha_{3}$ such that $w\left(l, i_{1}, j_{1}\right)=\alpha_{1}$ for all $l \in \mathcal{N} \backslash\left\{i_{1}, j_{1}\right\}, w\left(l, i_{2}, j_{2}\right)=\alpha_{2}$ and $w\left(l, j_{2}, k_{2}\right)=\alpha_{3}$ for all $l \in \mathcal{N} \backslash\left\{i_{2}, j_{2}, k_{2}\right\}, w\left(i_{2}, j_{2}, k_{2}\right)=\alpha_{2}+\alpha_{3}$, and all other triads are consistent.

3. There are edges $(i, j),(j, k),(k, s)$, where $|\{i, j, k, s\}|=4$, and nonzero values $\alpha_{1}, \alpha_{2}, \alpha_{3}$ such that $w(l, i, j)=\alpha_{1}$ for all $l \in \mathcal{N} \backslash\{i, j, k\}, w(l, j, k)=\alpha_{2}$ for all $l \in \mathcal{N} \backslash\{i, j, k, s\}, w(l, k, s)=\alpha_{3}$ for all $l \in \mathcal{N} \backslash\{j, k, s\}, w(i, j, k)=\alpha_{1}+\alpha_{2}$, $w(j, k, s)=\alpha_{2}+\alpha_{3}$, and all other triads are consistent.

4. There is a triad $(i, j, k)$ and nonzero values $\alpha_{1}, \alpha_{2}, \alpha_{3}$ such that $w(l, i, j)=\alpha_{1}$, $w(l, j, k)=\alpha_{2}$ and $w(l, k, i)=\alpha_{3}$ for all $l \in \mathcal{N} \backslash\{i, j, k\}, w(i, j, k)=\alpha_{1}+\alpha_{2}+\alpha_{3}$, and all other triads are consistent.

5. There are different edges $(i, j),(i, k),(i, s)$ and nonzero values $\alpha_{1}, \alpha_{2}, \alpha_{3}$ such that $w(l, i, j)=\alpha_{1}, w(l, i, k)=\alpha_{2}$ and $w(l, i, s)=\alpha_{3}$ for all $l \in \mathcal{N} \backslash\{i, j, k, s\}$, $w(i, j, k)=\alpha_{1}-\alpha_{2}, w(i, k, s)=\alpha_{2}-\alpha_{3}, w(i, s, j)=\alpha_{3}-\alpha_{1}$, and all other triads are consistent.

For any triple of edges fulfilling one of the conditions 1 through 5 , the modification of the weights of the edges that makes the graph $G$ consistent is unique except for condition 4 in case of $n=3$, and condition 5 in case of $n=4$, when there is and there 
may be, respectively, an infinite number of possible modifications. If $n \geq 6$, then at most one of conditions 1 through 5 holds, furthermore, there exists at most one triple of edges fulfilling any of conditions 1 through 4 . If $n \geq 8$, then there exists at most one triple of edges fulfilling condition 5 .

Proof: First, assume that $A$ can be made consistent by modifying three elements. It is easy to see that the disposition of the corresponding edges is isomorphic to one of those in Figure 7. We can also assume that the direction of the edges are the same as in Figure 7 since taking the reciprocal position of an element in $A$ means changing the direction of the corresponding edge in $G$.

If the three edges are arranged as in Figure $7(\mathrm{a})$, and in order to make $A$ consistent a nonzero $\beta_{t}$ is added to $\bar{a}_{i_{t} j_{t}}, t=1,2,3$, then the weight of $\left(l, i_{t}, j_{t}\right)$ is modified by adding $\beta_{t}$ to it for all $l \in \mathcal{N} \backslash\left\{i_{t}, j_{t}\right\}, t=1,2,3$. Since the weight of any triad is zero in the modified graph, it is evident that condition 1 holds with $\alpha_{t}=-\beta_{t}, t=1,2,3$.

If the three edges are arranged as in Figure 7(b), and in order to make $A$ consistent nonzero $\beta_{1}, \beta_{2}$ and $\beta_{3}$ are added to $\bar{a}_{i_{1} j_{1}}, \bar{a}_{i_{2} j_{2}}$ and $\bar{a}_{j_{2} k_{2}}$, respectively, then it is easy to see that condition 2 holds with $\alpha_{t}=-\beta_{t}, t=1,2,3$.

The proof of necessity for the disposals in Figures 7(c)-(e) works in a similar way as above, so we leave it to the reader.

The sufficiency is also easy to see. If condition 1 holds, we add $-\alpha_{t}$ to $\bar{a}_{i_{t} j_{t}}, t=$ $1,2,3$, and all triads become consistent. If condition 2 holds, we add $-\alpha_{1},-\alpha_{2}$ and $-\alpha_{3}$ to $\bar{a}_{i_{1} j_{1}}, \bar{a}_{i_{2} j_{2}}$ and $\bar{a}_{j_{2} k_{2}}$, respectively. If condition 3 holds, $-\alpha_{1},-\alpha_{2}$ and $-\alpha_{3}$ are added to $\bar{a}_{i j}, \bar{a}_{j k}$ and $\bar{a}_{k s}$, respectively. In the cases of conditions 1 and 2 , the modification of the weights of the edges that makes $G$ consistent is unique since for any of the three edges there is always a triad constructed without the other two edges, and this triad determines the modification unambiguously. In the case of condition 3 , the uniqueness of the modifications of the weights of the edges $(i, j)$ and $(k, s)$ comes from $w(s, i, j)=\alpha_{1}$ and $w(i, k, s)=\alpha_{3}$, respectively, and that of edge $(j, k)$ follows from $w(i, j, k)=\alpha_{1}+\alpha_{2}$ and $w(j, k, s)=\alpha_{2}+\alpha_{3}$.

In the case of condition 4 and $n=3$, we can modify the weight of two edges arbitrarily, this determines unambiguously how to modify the weight of the third edge to make $G$ consistent. If $n \geq 4$, the proof of the modification of the weights of the edges and its uniqueness is the same as for conditions 1 and 2 .

In the case of condition 5 and $n=4$, the modification of the weights of the edges may not be unique. For example, if we have $\bar{a}_{j k}=\bar{a}_{k s}=\bar{a}_{s j}=0$, we can choose an $\alpha$ different from the weights of $(i, j),(i, k)$ and $(i, s)$ arbitrarily, and change the weights of these edges to $\alpha$. Then the graph $G$ becomes consistent. However, if $n \geq 5$, then the modification is unique, and it can be proved in the same way as for conditions 1 and 2 .

Finally, we show that if $n \geq 6$ and a triple of edges fulfils any of conditions 1 through 4 , or $n \geq 8$ and a triple of edges fulfills condition 5 , then there exists no other triple of edges fulfilling any of conditions 1 through 5 . This proves the last statements of the proposition directly. The technique of proof is simple. We consider a triple of edges fulfilling one of conditions 1 through 5 , and assume contrarily that there exists another triple of edges fulfilling any of condition 1 through 5 , offering another way to make $G$ consistent. We show that this assumption leads to a contradiction.

If condition 1 holds for the first triple of edges, we can assume without loss of generality that the first triple is the one constructed in the proof of sufficiency, i.e. the one in Figure $7(\mathrm{a})$, furthermore, the edge $\left(i_{1}, j_{1}\right)$ is in the first triple but not in the second one. However, the four triads $\left(l, i_{1}, j_{1}\right)$, where $l \in\left\{i_{2}, j_{2}, i_{3}, j_{3}\right\}$, are inconsistent, 
and they cannot be made consistent by modifying only the weights of the edges of the second triple.

Assume now that condition 2 holds for the first triple of edges constructed in the proof of sufficiency, i.e. for the one in Figure $7(\mathrm{~b})$, and again that $\left(i_{1}, j_{1}\right)$ is in the first triple but not in the second one. Since $n \geq 6$, there exists a node $l$ different from the nodes of the edges in the first triple. The four triads $\left(t, i_{1}, j_{1}\right)$, where $t \in\left\{l, i_{2}, j_{2}, k_{2}\right\}$, are inconsistent, and cannot be made consistent by modifying only the weights of the second triple. If $\left(i_{1}, j_{1}\right)$ is in the second triple but, say, $\left(i_{2}, j_{2}\right)$ is not, then the three inconsistent triads $\left(l, i_{2}, j_{2}\right),\left(i_{1}, i_{2}, j_{2}\right)$ and $\left(j_{1}, i_{2}, j_{2}\right)$ cannot be made consistent by modifying only the weights of the two other edges of the second triple.

Assume now that condition 3 holds for the first triple of edges constructed in the proof of sufficiency, i.e. for the one in Figure $7(\mathrm{c})$. Since $n \geq 6$, there exist nodes $l_{1} \neq l_{2}$ different from $i, j, k$ and $s$. We show that at least one of $(i, j)$ and $(k, s)$ must be in the second triple. If contrarily the weight of neither $(i, j)$ nor $(k, s)$ is modified, then the four inconsistent triads $\left(l_{1}, i, j\right),\left(l_{2}, i, j\right),\left(l_{1}, k, s\right)$ and $\left(l_{2}, k, s\right)$ cannot be made consistent by modifying the weights of three edges. Thus, without loss of generality, we can assume that $(i, j)$ is in the second triple. Since $w(t, i, j)=\alpha_{1}$ for all $t \in\left\{l_{1}, l_{2}, s\right\}$, the weight of $(i, j)$ is to be modified, and the weight of at most two further edges can be modified, it follows immediately that the only way to make these three triads consistent is that we add $\beta_{1}=-\alpha_{1}$ to the weight of $(i, j)$. After this modification we have $w(t, j, k)=\alpha_{2}$ for all $t \in\left\{i, l_{1}, l_{2}\right\}$, and the only way to make these triads consistent by modifying the weight of at most two further edges is that we add $\beta_{2}=-\alpha_{2}$ to the weight of $(j, k)$. In the same way, we get also that $\beta_{3}=-\alpha_{3}$ is to be added to the weight of $(k, s)$. This means that the second triple is just the first one.

Assume now that condition 4 holds for the first triple of edges constructed in the proof of sufficiency, i.e. for the one in Figure $7(\mathrm{~d})$. Since $n \geq 6$, there exist different nodes $l_{1}, l_{2}$ and $l_{3}$ different also from $i, j$ and $k$. Assume that the edge $(i, j)$ is in the first triple, but is not in the second one. Since the three triads $(t, i, j), t \in\left\{l_{1}, l_{2}, l_{3}\right\}$, are inconsistent, the weight of either $(t, i)$ or $(t, j)$ is to be modified for all $t \in\left\{l_{1}, l_{2}, l_{3}\right\}$. Then, however, the weight of $(j, k)$ and $(k, i)$ cannot be modified. Since there is no way to modify the weight of any further edge, if the weight of $\left(l_{1}, i\right)$ is modified, then $\left(l_{1}, j, k\right)$ remains inconsistent, similarly, if the weight of $\left(l_{1}, j\right)$ is modified, then the same holds for $\left(l_{1}, i, k\right)$.

Assume, finally, that condition 5 holds for the first triple of edges constructed in the proof of sufficiency, i.e. for the one in Figure $7(\mathrm{e})$. Let $n \geq 8$, then there exist different nodes $l_{1}, l_{2}, l_{3}$ and $l_{4}$ different also from $i, j, k$ and $s$. Assume, without loss of generality, that $(i, j)$ is not in the second triple of edges. Then the four triads constructed from $(i, j)$ and one of $l_{1}, l_{2}, l_{3}$ and $l_{4}$ are inconsistent, and they cannot be made consistent by modifying the weights of at most three edges.

Remark 5. For the cases not addressed in Proposition 7, numerical examples can be easily constructed similarly to those in Remarks 2 and 3. It can be shown that if $n \leq 5$, then more than one of conditions 1 through 5 can simultaneously hold, furthermore, for any of conditions 1 through 4 an example with more than one triple fulfilling the condition can be constructed. The same holds for $n \leq 7$ and condition 5 . The constructions are left to the reader.

Remark 6. To perform the operations according to Proposition 7, we have to prepare the list of the inconsistent triads with $O\left(n^{3}\right)$ operations. If the number of the inconsistent triads is less than $3(n-2)-6$ or greater than $3(n-2)$, then it is sure that 
there is not any triple of edges fulfilling any of conditions 1 through 5 of Proposition 7. Otherwise, from the list of the edges appearing in the list of the inconsistent triads, we can prepare a list of $O\left(n^{3}\right)$ triples of edges as candidates to fulfill any of conditions 1 through 5. For each of these triples, we have to identify the disposition of the edges according to Figure 7 as well as the relating condition of those listed in Proposition 7, and then to check the relating condition. This can be done with $O(n)$ operations. To check that only triads based on at least one edge of the triple can be found in the list of the inconsistent triads, $O(n)$ further operations are needed. Altogether, the triples of edges fulfilling any of conditions 1 through 5 , and how to modify can be determined with $O\left(n^{4}\right)$ operations. Even if the number of the inconsistent triads is between $3(n-2)-6$ and $3(n-2)$, it may happen that there is not any triple of edges fulfilling at least one of conditions 1 through 5 of Proposition 7 . This is the situation, for example, if in matrix $A$ of Example 2 we change $a_{1,2}$ to 9 and $a_{2,1}$ to $1 / 9$.

\section{Empirical pairwise comparison matrices}

In order to see the relevance of the results of Sections 2 through 6 it is essential to make test on matrices originated from real decision problems. The idea of analysing empirical pairwise comparison matrices comes from the studies of Gass, Standard [12, 6]. An on-line collection of empirical pairwise comparison matrices (EPCM,[10]) offers the opportunity of any kind of analysis or test that may be interesting from both theoretic and practical points of view. The collection is growing and it is open to everyone. Each matrix is originated from a paper, typically a case study, published in well-known journals of MADM and related topics. Matrices are available in different formats like Excel, Matlab or PDF. New matrices with the corresponding citations are also welcome in the list. The aim is to develop an open database of a proper size from which one gets a better view of the nature of real problems.

Our analysis has been made based on EPCM-October-2009 consisting from 137 empirical matrices. The above mentioned matrices came from 22 different decision problems which were published in scientific journals. See [10] for full citations. We have got the following table by solving the mixed 0-1 programming problem (2) in MATLAB. The inconsistent pairwise comparison matrices that can be made consistent by modifying a few (1-3) of its elements can be detected by the tools presented in Section 2. It is also counted how often these cases occur in real/empirical decision problems (Table 1).

\begin{tabular}{|l|c|c|c|c|c|}
\hline Dimension & $\begin{array}{c}\text { Number of } \\
\text { matrices }\end{array}$ & Consistent & $\begin{array}{c}1 \text { element } \\
\text { to modify }\end{array}$ & $\begin{array}{c}2 \text { elements } \\
\text { to modify }\end{array}$ & $\begin{array}{c}3 \text { elements } \\
\text { to modify }\end{array}$ \\
\hline $3 \times 3$ & 30 & 14 & 16 & - & - \\
\hline $4 \times 4$ & 20 & 1 & 6 & 7 & 0 \\
\hline $5 \times 5$ & 19 & 1 & 1 & 5 & 1 \\
\hline $6 \times 6$ & 21 & 0 & 1 & 1 & 0 \\
\hline $\begin{array}{l}7 \times 7 \\
\text { and larger }\end{array}$ & 47 & 0 & 1 & 0 & 0 \\
\hline
\end{tabular}

Table 1 The number of consistent matrices and of the ones that can be made consistent by the modification of a few elements among the 137 empirical pairwise comparison matrices 


\section{References}

1. Bozóki, S, Lewis, R (2005): Solving the Least Squares Method problem in the AHP for $3 \times 3$ and $4 \times 4$ matrices. Central European Journal of Operations Research 13: 255-270.

2. Bozóki, S, Rapcsák, T (2008) On Saaty's and Koczkodaj's inconsistencies of pairwise comparison matrices. Journal of Global Optimalization 42(2): 139-148

3. Bozóki S (2008) Solution of the least squares method problem of pairwise comparisons matrices. Central European Journal of Operations Research 16: 345-358

4. Chu, ATW, Kalaba, RE, Spingarn, K (1979) A comparison of two methods for determining the weight belonging to fuzzy sets. Journal of Optimization Theory and Applications 4: $531-538$

5. Gass, SI (1998) Tournaments, transitivity and pairwise comparison matrices. Journal of the Operational Research Society 49: 616-624

6. Gass, SI, Standard, SM (2002) Characteristics of positive reciprocal matrices in the analytic hierarchy process. Journal of Operational Research Society 53: 1385-1389

7. Kéri, G (2005) Criteria for pairwise comparison matrices. Szigma 36: 139-148 (in Hungarian)

8. Koczkodaj, WW (1993) A new definition of consistency of pairwise comparisons. Mathematical and Computer Modelling 18: 79-84.

9. Poesz, A (2008) Analysis of the inconsistency of empirical pairwise comparison matrices. Master's Thesis, Department of Decisions in Economics, Corvinus University of Budapest.

10. Poesz, A (2009) Empirical pairwise comparison matrices (EPCM) - an on-line collection from real decisions, version EPCM-October-2009. http://www.sztaki.hu/ bozoki/epcm

11. Saaty, TL (1980) The analytic hierarchy process. McGraw-Hill, New-York.

12. Standard, SM (2000) Analysis of positive reciprocal matrices. Master's Thesis, Graduate School of the University of Maryland. 